\title{
Norois
}

Environnement, aménagement, société

$217 \mid 2010 / 4$

Interroger les processus de valorisation des espaces urbains

\section{Introduction : De l'appropriation à la valorisation, et retour}

Maria Gravari-Barbas et Fabrice Ripoll

\section{(2) OpenEdition \\ Journals}

Édition électronique

URL : http://journals.openedition.org/norois/3435

DOI : 10.4000 /norois.3435

ISBN : 978-2-7535-1565-9

ISSN : $1760-8546$

\section{Éditeur}

Presses universitaires de Rennes

\section{Édition imprimée}

Date de publication : 15 décembre 2010

Pagination : 7-12

ISBN : 978-2-7535-1340-2

ISSN : 0029-182X

\section{Référence électronique}

Maria Gravari-Barbas et Fabrice Ripoll, «Introduction : De l'appropriation à la valorisation, et retour », Norois [En ligne], 217 | 2010/4, mis en ligne le 22 février 2011, consulté le 22 septembre 2020. URL : http://journals.openedition.org/norois/3435; DOI : https://doi.org/10.4000/norois.3435 


\title{
Introduction
}

\section{DE L'APPROPRIATION À LA VALORISATION, ET RETOUR}

\author{
Maria Gravari-Barbas \\ EA EIREST \\ (Université de Paris 1 Panthéon Sorbonne), \\ 12, place du Panthéon - 75005 PARIs, France \\ maria.gravari-barbas@wanadoo.fr \\ FABRice RipolL \\ EA Lab’Urba \\ (Université Paris Est Créteil), \\ 61, avenue du Général-de-Gaulle - 94010 Créteil cedex, France \\ fabrice.ripoll@u-pec.fr
}

\section{De l'appropriation à la valorisation}

Il y a quelques temps déjà, dans cette même revue, fut défendue l'idée que la question de l'appropriation de l'espace était incontournable voire centrale en géographie, et plus largement en sciences sociales (Ripoll et Veschambre, 2005). Et cela pour de nombreuses raisons : à la fois conceptuelles, problématiques et théoriques. À l'époque, la notion de territoire et ses dérivés faisaient sérieusement fureur dans la discipline, sans que leur principal fondement sémantique soit interrogé. Il fallait donc, a minima, sortir de cette situation, pour le moins paradoxale et injustifiable, en dégageant les différents usages de l'expression. Plus encore, quels que soient ces usages, revenir au champ lexical de l'appropriation permettait de contrer la tendance, encore forte dans la discipline, consistant à préférer les formes spatiales ou « objets géographiques » (territoire, ville, région, etc.) aux processus et rapports sociaux. Au risque de la réification ou du fétichisme : penser que, ou faire comme si ces formes existaient en elles-mêmes, postuler leur existence, c'est projeter sur le sol et figer des pratiques et représentations sociales changeantes, diverses, voire contradictoires. Ce qui ne veut pas dire que l'espace matériel n'a pas une existence indépendante et contraignante, ni que les corps n'en font pas partie!

Bien entendu, une telle notion avait aussi l'avantage de nous empêcher d'oublier les inégalités sociales et les rapports de pouvoir, et permettait de montrer qu'ils se nichaient au sein même de ces rapports à l'espace matériel, traversant les sociétés à toutes les échelles. De ce point de vue, c'est d'abord la tendance à considérer que les collectivités humaines ou les groupes sociaux s'approprient « comme un seul homme » des portions d'espace, elles-mêmes juxtaposées les unes à côté des autres comme dans une mosaïque, qui se trouvait mise en question. Ces rhétoriques holistes, véhiculées dans les discours territoriaux (identitaires et communautaristes) des acteurs eux-mêmes, devaient être prises pour objet de recherche sans les reprendre à notre compte. Mais il ne s'agit pas pour autant d'adopter la posture opposée consistant à déclarer les territoires obsolètes voire pathologiques pour mieux mettre l'accent sur les formes en réseau, tout aussi fétichisées, et/ou sur une mobilité prétendument généralisée et d'autant plus facilement assimilée à la liberté qu'elle s'étend à un espace plus vaste. 
Maria Gravari-Barbas, Fabrice Ripoll

Comment considérer le Monde comme ouvert à tous et à toutes les possibilités, quand on regarde en face les enfermements, assignations et rétentions, les contrôles de déplacements, les inégalités d'accès au sein même des espaces les plus «publics » des villes les plus « cosmopolites » des sociétés les plus «démocratiques »?

Certes, des espaces sont appropriés par les plus démunis, pour ne pas dire les plus dominés, mais peut-on sérieusement affirmer qu'ils sont de même valeur que les autres? Pointer les ségrégations ne prend tout son sens que lorsqu'on articule la séparation géographique avec l'inégalité de valeur des lieux habités. N'est-ce pas le même genre de constats que l'on peut faire quant aux autres lieux fréquentés (pour le travail, les loisirs, etc.) ? A la hiérarchie sociale correspond, grosso modo, une hiérarchie des espaces assignés et/ou appropriés. Mais qu'en est-il des biens ou espaces a priori partagés, communs, ou tout au moins accessibles à toutes et tous? Aborder de front la question de l'appropriation a permis d'approfondir une réflexion collective engagée depuis plusieurs années sur le patrimoine. Qui dit patrimoine dit objet, monument, espace, etc., d'une valeur supérieure pour ceux qui se reconnaissent en lui. Qui dit patrimoine dit aussi référence partagée, identité et mémoire collectives, bien commun. Mais peut-on réellement considérer qu'il y a toujours et partout appropriation collective? Comment se fait-il, dans ce cas, que le patrimoine puisse susciter tant de conflits (Grasnier, 2000) ? Et même dans les cas où le patrimoine fait consensus, cela ne pas veut pas dire qu'il apporte une jouissance et des profits équivalents à tout un chacun. C'est qu'un patrimoine est aussi une forme de marquage social de l'espace (Bulot et Veschambre, 2006) : outre sa mise à distance ou son inaccessibilité, c'est sa reconnaissance même, en tant qu'ode à la grandeur des puissants, qui peut être assimilée à de la violence symbolique. De même que sa figure inversée : la démolition du bâti stigmatisé, véritable effacement des traces des populations indésirables (Veschambre, 2008). Cette question de la valeur, que l'analyse du patrimoine permet de mettre en avant, est donc inséparable de celle de l'appropriation, et tout aussi incontournable qu'elle.

Mais les choses se compliquent, et de plusieurs manières. D’une part, on ne peut se contenter de parler de la valeur des espaces sans déconstruire cette notion, sans interroger son contenu. Or, le fait est qu'il existe de nombreux critères ou types de valeurs. A n'en pas douter, le couple valeur d'usage/valeur d'échange légué par la tradition marxiste, dans sa version lefebvrienne (Lefebvre, 1974, 2000), reste une grille d'analyse pertinente des sociétés contemporaines. Elle fonde l'analyse des processus de privatisation des espaces publics et plus encore de marchandisation, à travers par exemple la mise en valeur touristique, le « marketing territorial » en contexte de concurrence généralisée (Gravari-Barbas, 2006). Mais aussi intéressant soit-il, il ne rend pas compte de la multiplicité des critères utilisés par les acteurs pour affirmer la valeur d'un lieu, et plus généralement la hiérarchie des espaces : économiques, fonctionnels, mais aussi esthétiques, symboliques, affectifs... Mais faire ce genre d'énumération pointe aussitôt une série de questions quant aux rapports qu'entretiennent ces critères d'appréciation. Sont-ils cumulatifs ou, au contraire, exclusifs et contradictoires? Ou encore, eux-mêmes hiérarchisés? Et le sont-ils de la même manière pour tout un chacun? Impossible en effet de négliger le fait que, des critères économiques dont le degré d'universalisation est tel qu'ils semblent échapper même à la volonté humaine, aux critères affectifs les plus subjectifs, ces formes de valeur ne connaissent pas le même degré d'objectivation. Mais dire cela, c'est souligner qu'à la notion de valeur doit être préférée celle de valorisation, comprise comme un processus social, qui constitue la raison d'être de ce dossier.

\section{La valorisation : un processus de construction sociale daté et localisé}

Deux ans après la sortie du numéro de Norois sur la question de l'appropriation de l'espace (Ripoll et Vexchambre, 2005) était organisé un colloque pluridisciplinaire pour l'articuler aux questions de catégorisation et de (dé)valorisation, et interroger ainsi les relations entre 
hiérarchie des espaces et inégalités sociales ${ }^{1}$. Outre que deux articles publiés ici en sont issus (ceux de Ter Minassian et Zanetti), l'ensemble du colloque fait l'objet d'une analyse détaillée par R. Keerle qui souligne à la fois les convergences, les points de discussion et les pistes pour approfondir la réflexion théorique sur ce qu’il appelle la «spatialisation de la valeur».

Pour nourrir cette réflexion, les études empiriques réunies dans ce numéro insistent sur la manière dont les objets sont investis de valeur, selon des logiques et en fonction de temporalités qui peuvent être cernées, analysées et explicitées. La «traçabilité » des processus de valorisation est possible car ceux-ci sont toujours localisés et datés. L'une des questions importantes, soulevée par les différents articles, est de comprendre précisément pourquoi la valorisation se produit à tel endroit et pas ailleurs, à tel moment et pas à un autre : comment le processus de valorisation d'un objet, d'un bâtiment, d'un site se met-il en marche? Qui l'active? Et pour quelles raisons?

Les articles insistent d'abord sur les contextes ou les événements qui représentent des bifurcations importantes dans la manière dont tel site ou objet est appréhendé par différents groupes sociaux, conjointement ou de manière divergente. Ainsi la temporalité de la «mise en marche » du processus de valorisation du «patrimoine Perret » au Havre (Gravari-Barbas et Renard), ou celle de la Manufacture d'Armes à Saint-Étienne (Zanetti), est cernée à la fois par l'intermédiaire du contexte général dans lequel elle s’inscrit (celui de la montée de la reconnaissance du patrimoine dans les sociétés occidentales), du contexte local (les ambitions de développement économique et de requalification d'espaces, promues par les acteurs locaux) et des événements éventuels pouvant accélérer le processus (menaces de disparition d'un élément en voie de patrimonialisation, qui fonctionne parfois comme un «multiplicateur de valeur»).

Outre l'analyse de la temporalité de leur enclenchement, les auteurs s'attachent au phasage des processus de constructions sociales productrices de valeurs. Les logiques sont rarement linéaires et simplement cumulatives : les processus de valorisation mis en marche à Barcelone (Ter Minassian) s'accompagnent ou, tout au moins, sont à lire conjointement avec des processus de dévalorisation. Les deux sont liés, sinon par des relations de cause à effet, du moins par des processus de transfert de valeurs aussi importants à saisir que les processus de création. Les auteurs montrent ainsi cet « avant » et cet « après » dans la constitution des valeurs, d'autant plus mythique que la dévalorisation préalable est ancienne, profonde, marquante, à la fois dans l'espace et dans les imaginaires. Comment la déqualification initiale est-elle dépassée pour permettre la revalorisation et l’investissement économique de quartiers marginaux? Comment « les mots qui semblaient ancrés, rivés au centre historique de Gênes ont-ils été balayés par de nouvelles appropriations des espaces? » (Jacquot).

La dévalorisation préalable des espaces (qu'elle soit objectivée, orchestrée, instrumentalisée ou fantasmée) permet, et légitime, l'entrée en scène de divers acteurs qui promeuvent leur (re)valorisation. Qu'elle soit présentée comme une urgence (Gênes, Barcelone), une nécessité (Le Havre) ou une opportunité (Saint-Etienne), les actions et les discours qui la justifient se nourrissent de, s'appuient sur, se mesurent et s'évaluent par rapport à ce constat d'une dévalorisation forcément déplorable. Les « entrepreneurs de (re)valorisation » adoptent, s'approprient ou produisent ainsi des argumentaires visant à rendre évidente la nécessité d'actions (ré)génératrices, produisant de nouvelles valeurs dont l'apport est d'autant plus important que l'état antérieur est dévalué. La valorisation se mesure en effet en termes de plus values (économiques, foncières, sociales, d'image, etc.) dont l'obtention, ne serait-ce que symbolique, est également nécessaire pour faire entrer les projets en cours dans un cercle vertueux, une spirale positive.

1. Le colloque, intitulé "Espaces hérités, espaces enjeux : appropriations - (dé)valorisations - catégorisations », s'est tenu à l'université de Caen Basse-Normandie les 8-9-10 novembre 2007. Il a été organisé par l’UMR CNRS ESO, les pôles pluridisciplinaires «Villes et sciences sociales » et «Sociétés et espaces ruraux » de la Maison de la Recherche en Sciences Humaines de l'université de Caen, le Programme de Recherches Interdisciplinaires «Études urbaines » de l'EHESS Paris, et le CREDILIF de l'université de Rennes 2. 


\section{Les échelles de référence des stratégies de valorisation : du local à l'international}

Les processus de valorisation sont ainsi enclenchés, accompagnés, soutenus (mais aussi contrecarrés, contestés, empêchés) par les acteurs sociaux en présence pour des raisons diverses qu'il est possible d'analyser et décoder, offrant une grille de lecture passionnante de la manière dont on produit, diachroniquement, la ville. La pluralité des acteurs présents sur la scène locale - les articles insistent en particulier sur l'opposition entre acteurs urbains et associations - pointe la possibilité de lectures différenciées des valeurs à produire. Les discours alors développés pour communiquer, créer du consensus, entraîner et mobiliser, offrent une matière première stimulante des représentations produites localement (dans chaque contexte) des valeurs et des plus-values possibles de ces espaces urbains en projet. Dans la plupart des cas étudiés, il s'agit de "raccrocher » des espaces, considérés comme « en perte de vitesse », aux transformations dont ils ont été exclus ou marginalisés. Or, le décalage à rattraper ne se mesure pas uniquement par rapport à des zones ou quartiers situés dans la même agglomération, mais aussi par rapport à des réalités extra-locales, nationales ou internationales.

Dans le cas des villes européennes étudiées (Saint-Étienne, Barcelone, Le Havre et Gênes), les articles mettent ainsi en évidence des systèmes d'acteurs, agissant concrètement sur le terrain ou prescrivant les valorisations à plusieurs nivaux scalaires. La préoccupation des acteurs urbains est, entre autres, de « mettre à l'heure » la pendule locale par rapport à l'heure mondiale. Les acteurs municipaux et urbains orchestrent souvent des projets de requalification d'espaces en friche, dévalorisés ou accusant un décalage par rapport à « ce qui se fait ailleurs », autrement dit en référence à un système de valeurs prescrit ou induit par un système-Monde synonyme de mise en concurrence généralisée. En ce sens, leur public cible n'est pas purement local : il est également celui des élites mobiles et transnationales (hommes d'affaires, touristes). Ceci pose d'emblée les objectifs, le processus et le « produit final » de la valorisation dans un cadre d'analyse différent de ceux qui se référent uniquement au contexte local.

Les projets de valorisation opèrent ainsi, de manière plus ou moins réussie, un « grand écart » entre la production de valeurs se référant aux attentes des populations locales et celles auxquelles aspirent les «classes créatives », cible généralisée des politiques de valorisation des espaces urbains - de manière plus ou moins explicite et avouée - au cours de la première décennie du XXI $^{\mathrm{e}}$ siècle. Si, par exemple, Saint-Étienne érige le design en tant qu'objectif principal des politiques urbaines stéphanoises, en s'appuyant pour cela sur une identité locale réinterprétée, c'est pour s'offrir un référentiel d'action global, susceptible de communiquer internationalement. Et c'est ce référentiel global qui, par un tour de passe-passe habile, sert de fondement à la patrimonialisation de la manufacture d'armes. Ce " grand écart » opère toutefois des sélections et des tris, en fonction du référentiel de valorisation esquissé, du cadre général dans lequel il opère et de ses publics cibles. Les éléments bâtis ou les espaces qui ne peuvent pas servir ce référentiel, ni ces publics cibles, sont ainsi sacrifiés. Dans le cas de Saint-Étienne, c'est le cas des bâtiments d'administration de la Manufacture - qui auraient probablement été sauvegardés si le référentiel de valorisation était fondé sur le patrimoine.

Cela dit, la patrimonialisation peut aussi être définie comme l'injection de valeurs symboliques s'accompagnant le plus souvent par une revalorisation économique. Et, en la matière, si les acteurs urbains se font experts dans le maniement des outils d'un marketing territorial instrumentalisant de plus en plus le patrimoine, leurs villes sont aussi de plus en plus observées par des regards experts internationaux à l'affut d'espaces ayant un potentiel patrimonial. Dans le cas du Havre, l'éveil du «patrimoine Perret », sa « mise en discours » ou la conception de la boite à outils qui a accompagné sa patrimonialisation, n'auraient pas été faits de la même manière et selon les mêmes temporalités sans l'intervention de ce « regard extérieur ». Toute la question réside dans la compréhension de qui est-ce qui décide/produit 
« la rentabilité du temps qui passe »(Carballo et Emeliannof, 2002), selon quelles logiques et via quels processus. C'est que la valorisation des espaces est suggérée et induite par des acteurs internationaux qui examinent le «potentiel » des espaces non pas à travers la grille de lecture locale - celle des représentations identitaires, aspirations, besoins fonctionnels et symboliques de la population - mais à travers un regard exogène : experts internationaux, conseillers, architectes, urbanistes et artistes bien insérés dans le système global, ONG, élites transnationales, hommes d'affaires, touristes, etc., qui contribuent à des degrés divers à cette « réinsertion » des espaces dans un monde globalisé en imposant leurs propres standards (esthétiques, culturels, économiques, symboliques, etc.).

Mais si la mondialisation éveille le local, on observe bien que l'éveil ne s'opère pas de la même manière partout : c'est donc que le local se réveille de manière différenciée... Et cette intégration dans le système-Monde n'est pas toujours le fruit des stratégies d'acteurs institutionnels. Elle peut se faire «par le bas » (Bergel et Kerdoud). Les développements commerciaux à Constantine donnent ainsi à voir des processus spécifiques : tandis que dans les quatre villes européennes, il s'agit de se positionner propulser dans le système-Monde par l'intermédiaire d'une captation des flux internationaux, en invitant notamment les populations mobiles internationales à « consommer » sur place la plus value symbolique produite par les projets de requalification (patrimoine, création, espaces urbains exclusifs), dans la périphérie des villes algériennes, il s'agit au contraire d'offrir les produits mondialisés à la consommation locale. Les promoteurs de ces centres commerciaux qui «vendent le monde » dans leurs étalages contribuent, sans que ce soit leur intention, à intégrer les quartiers périphériques des villes algériennes dans l'espace mondialisé. Ces « Dubaï », fusion inédite entre shopping mall américain et références moyenorientales, non seulement produisent des valeurs inscrites dans les espaces dévalorisés des périphéries urbaines algériennes, mais participent de processus sociaux (genrés, culturels) dont les retombées, plus difficiles à analyser, constituent une piste intéressante des travaux à venir.

\section{Des situations complexifiées, des processus inachevés et potentiellement... inappropriés}

Dans ce jeu d'acteurs de la valorisation des espaces, les acteurs institutionnels urbains (villes, agglomérations, chambres de commerce, etc.) sont ainsi des acteurs-clé car ils apparaissent comme les principaux énonciateurs de stratégies très élaborées - en opposition tant avec les prescriptions des acteurs globaux qu'avec les actions associatives locales souvent purement réactives. Mais ils ne sont pas les seuls acteurs de la ville. Les stratégies qu'ils mettent en œuvre n'ont pas toujours les effets escomptés, les processus ne sont pas toujours aboutis et n'entraînent pas toujours la ville et sa population dans son ensemble.

Ces stratégies matérielles et idéelles de valorisation des espaces ne sont pas toujours construites de manière linéaire et continue : elles témoignent d'interrogations, de tâtonnements, de boucles d'action et de réaction. Elles n'aboutissent d'ailleurs pas toujours. Et c'est ici que réside toute la difficulté de l'analyse, dans l'évaluation nécessaire mais toujours imparfaite des processus de valorisation entrepris. Le cas de Barcelone (Ter Minassian) est emblématique d'une ville qui a réussi son intégration dans l'imaginaire global et qui projette internationalement une image rayonnante. Sa fulgurante évolution touristique est symptomatique de cette stratégie de "place making » et de «city branding ». Mais dans ce patchwork métropolitain barcelonais, les réalités sont diverses et contrastées : malgré cette euphorie amorcée dans les années 1990, certains morceaux de ville sont loin d'avoir réussi à "s'en sortir », d'autres se sont même marginalisés. Or, si le rôle du marketing territorial est d'attirer l'attention internationale sur les espaces valorisés promus au titre de nouvelles vitrines de la ville, le rôle du chercheur est de s'interroger sur l'ensemble de la ville, permettant des lectures plus nuancées de sa « réussite » et évitant des équations faussement valorisantes qui ne retiennent que les « bons » morceaux de ce patchwork urbain... 
Dans le cas du Vieux-centre de Gênes, S. Jacquot a également mis en évidence ce processus de valorisation et d'appropriation non abouti, visant dans un premier temps la création d'un modus vivendi accepté, sinon partagé, par les multiples occupants de l'espace. C'est un processus de « désappropriation » qui est ici mis en œuvre, par l'intermédiaire d'un contrôle des espaces dont le but est, toutefois, d'assurer l'appropriation future par des nouveaux usagers, touristes ou gentrifieurs. L'exemple des périphéries des villes algériennes apporte encore un regard différent. Contrairement aux quatre villes européennes, examinées essentiellement à travers les politiques mises en place par les municipalités, P. Bergel et N. Kerdoud montrent dans leur étude que les processus de valorisation peuvent faire l'objet d'actions non planifiées, non concertées, et parfois non autorisées! Ce qui redonne une place centrale aux acteurs non institutionnels. Mais il faut sans doute pousser les analyses encore plus loin.

Les articles réunis dans ce volume opposent souvent les résidents locaux « anciens » aux acteurs institutionnels (municipalité, agglomération, ou autres acteurs publics initiant les projets de requalification) d'une part, et aux groupes exogènes, en particulier touristiques, d'autre part. Or, les limites de la validité de ces oppositions méritent d'être questionnées. Dans les villes européennes étudiées, et sans doute davantage dans le cas de Barcelone, «l'ouverture » internationale spectaculaire de la ville au cours des deux dernières décennies a démultiplié les types d'acteurs et donc les modes d'appréciation de l'espace : entre habitants permanents des quartiers centraux, étudiants, hommes d'affaires, touristes nationaux ou internationaux parfois habitués, la lecture des processus d'appropriation en devient d'autant plus complexe. Si la valorisation des espaces par des stratégies d'acteurs publics ou de groupes privés (moins étudiés dans ce volume) contribue à enclencher et à faciliter les processus appropriatifs par des nouveaux venus constituant les publics ciblés, il serait fécond d'étudier plus directement les pratiques effectives de l'ensemble des acteurs et usagers. Peu d'études abordent ainsi les stratégies d'appropriation de l'espace par les résidents locaux (plus anciens ou nouveaux venus) face aux populations de passage, vues dans leur diversité (travailleurs émigrés, étudiants, touristes, mobilités d'affaires). Les a priori sur la légitimité de telle ou telle population dans un espace donné privent parfois l'analyse d'une lecture fine et nuancée, non prescriptive mais insistant sur les modalités diverses et parfois contradictoires des appropriations en cours.

\section{Bibliographie}

Bulot T., Veschambre V. (dir.), 2006. Mots, traces et marques : dimensions spatiale et linguistique de la mémoire urbaine, Paris, L'Harmattan, coll. «Espaces discursifs », 246 p.

Carballo C., Emeliannof C., 2002. La liquidation du patrimoine ou la rentabilité du temps qui passe, Annales de la recherche urbaine, $\mathrm{n}^{\circ}$ 92, p. 49-57.

Gravari-Barbas M., 2006. La ville à l'ère du loisir globalisé, Cahiers des Espaces, nº 2374, p. 48-56.

Lefebvre H., 1974. Le Droit à la ville, suivi de Espace et politique, Anthropos, Paris, coll. «Points », 284 p.

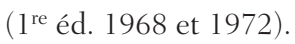

Lefebvre H., 2000. La Production de l'espace, Anthropos, Paris, coll. "Ethno-sociologie », 487 p. (1 re éd. 1974).

Gasnier A. (dir.), 2000. «Patrimoine et environnement. Les territoires du conflit », Norois, n ${ }^{\circ}$ 185, 173 p.

Ripoll F., Veschambre V. (dir.), 2005 : «L'appropriation de l'espace. Sur la dimension spatiale des inégalités sociales et des rapports de pouvoir », Norois, n 195, $117 \mathrm{p}$.

Veschambre V., 2008. Traces et mémoires urbaines. Enjeux sociaux de la patrimonialisation et de la démolition, PUR, Rennes, coll. « Géographie sociale », 315 p. 